\title{
Analysis of the willingness of DDKT candidates registered at a single center according to the Korean Kidney Donor Profile Index application
}

\author{
Saerom Lee ${ }^{1}$, Jung Ja Hong ${ }^{1}$, In Ok Kim${ }^{1}$, Ah Young Lee ${ }^{1}$, Ji Won Woo ${ }^{1}$, Seon Bin Park ${ }^{1}$, Shin Hwang ${ }^{2}$, Young Hoon Kim² \\ ${ }^{1}$ Organ Transplantation Center, Asan Medical Center, University of Ulsan College of Medicine, Seoul, Korea \\ ${ }^{2}$ Department of Surgery, Asan Medical Center, University of Ulsan College of Medicine, Seoul, Korea
}

Background: Kidney transplantation is the most ideal treatment method recommended for end-stage renal disease patients; since the establishment of Korean Network for Organ Sharing (KONOS) in 2000, the number of registered candidates on the waiting list for deceased donor kidney transplantation (DDKT) has grown to 27,062 as of December 2020 , increasing by $9 \%-10 \%$ every year. Due to the shortage of deceased donor to meet the growing demand, however, only about 800 DDKTs are being performed each year; consequently, the waiting period for transplantation has been also increasing every year, and as of the end of 2019, it has reached 2,196 days, an average of more than 6 years. With the recent increase in deceased donors with advanced age and/or complex diseases, it has become necessary for extended criteria donor (ECD) to be clearly specified. In March 2021, KONOS has formally defined ECD as a donor with a Korean Kidney Donor Profile Index (K-KDPI) 70\% or higher kidney in order to choose a suitable candidate of such in a more efficient manner. KONOS has also revised its guideline, in which, patients who do not consent to receive an ECD kidney to be removed from the list of candidates when matching an ECD kidney, effective September 15,2021 . Thus, the purpose of this study was to understand the willingness of DDKT candidates registered in a single center, and use the data as a basis of selection management of candidates when an ECD kidney becomes available.

Methods: We retrospectively studied 1,755 DDKT candidates registered at a single center as of March 15, 2021, via in-person and telephonic consultations, and online surveys from April to July 2021 to learn the willingness to accept a kidney from ECD.

Results: Of the 1,755 surveyed, 1,665 responded; the sex of respondents was 976 males (58.6\%) and 689 females (41.4\%); blood type was type $A(573,34.4 \%)$, type $B(463,27.8 \%)$, type $O(454,27.3 \%)$, and type $A B(175,10.5 \%)$; age was $0-9$ years old $(3,0.2 \%)$, $10-19$ years old $(8,0.5 \%), 20-29$ years old $(42,2.5 \%), 30-39$ years old $(164,39.9 \%), 40-49$ years old $(397,23.8 \%), 50-59$ years old $(580,34.8 \%), 60-69$ years old $(425,25.5 \%)$, and over 70 years old $(46,2.8 \%)$. Three hundred and seven candidates $(18.4 \%)$ were waiting for re-transplantation and six candidates $(0.4 \%)$ were past kidney donors. Of the 749 candidates $(45.3 \%)$ consented to accept an ECD kidney, 462 were males (61.7\%) and 287 were females (38.3\%); blood type of 253 patients (33.8\%) was type A, 208 patients $(27.8 \%)$ of type B, 213 patients $(28.4 \%)$ of type 0,75 patients $(10 \%)$ of type $A B ; 142$ patients $(19 \%)$ waiting for re-transplantation, and four patients ( $0.5 \%)$ of past kidney donor. The age group was 2 minors $(0.3 \%), 18(2.4 \%)$ people aged 20 to $29,65(8.7 \%)$ people aged 30 to 39, 159 (21.2\%) people aged 40 to 49, 250 (33.4) people aged 50 to 59, 227 (30.3\%) people aged 60 to 69 , and $28(3.7 \%)$ people aged 70 or older, with an average age of 53.5 years, a minimum age of 12 years, and a maximum age of 79.

Conclusions: $45.3 \%$ of those waiting for kidney transplants registered in a single center consented to accept an ECD kidney. It was confirmed that the patients with shorter waiting period and older age were more willing than the others. This result matched with the previous study done at this same center in 2020, in which willingness to accept a kidney from the elderly donors aged 60 or older with s-Cr>3.0 mg/dL or CRRT, or donors with $\mathrm{Cr}<1.5 \mathrm{mg} / \mathrm{dL}$ but have hypertension and diabetes. We expect this study to be an opportunity to provide waiting candidates with information and management prior to transplantation, and baseline data for medical staff to efficiently identify a suitable candidate when an ECD kidney becomes available.

Corresponding author: Saerom Lee

E-mail: hiromrom@gmail.com

(c) The Korean Society for Transplantation

This is an Open Access article distributed under the terms of the Creative Commons Attribution Non-Commercial License (http://creativecommons.org/licenses/by-nc/4.0/) which permits unrestricted non-commercial use, distribution, and reproduction in any medium, provided the original work is properly cited. 